\title{
GENUS TWO HEEGAARD SPLITTINGS
}

\author{
JOEL HASS
}

(Communicated by Frederick R. Cohen)

\begin{abstract}
It is shown that a 3-manifold has a finite number of genus two Heegaard splittings. A corollary is that the mapping class group is finite if the manifold is non-Haken.
\end{abstract}

This paper explores a relation between a rigidity property in the theory of minimal surfaces and the problem of classifying Heegaard surfaces and diffeomorphisms of 3-dimensional manifolds.

Theorem 4 states that a closed 3-manifold has a finite number of genus two Heegaard splittings up to homeomorphism. This result contrasts with a recent result of Sakuma, who found a closed 3-manifold that possesses an infinite number of genus two Heegaard splittings, distinct up to isotopy [S]. In fact, Sakuma found infinitely many such manifolds. Since genus two manifolds have geometric decompositions [Th2] and the genus two Seifert fiber spaces and connect sums of lens spaces are known to have only finitely many distinct Heegaard splittings of genus two, it is a consequence of Theorem 4 that only manifolds with nontrivial torus decompositions can have an infinite number of genus two Heegaard splittings that are distinct up to isotopy. By combining Theorem 4 with some recent work of Hass-Scott [HS], we obtain Corollary 8, which states that the mapping class group of a non-Haken irreducible 3-manifold of genus two is finite.

A minimal embedding of a 2-dimensional surface in a Riemannian 3-manifold is an embedding with mean curvature zero. There is no known oneparameter family of minimal embeddings in a negative curvature 3-manifold, and it is reasonable to guess that the space of minimal embeddings of a given genus surface is finite. This is true for a generic metric of negative curvature by results of White [Wh], since if false, there would exist in $M$ a minimal embedding that has a nontrivial Jacobi field, and White's result rules this out for generic metrics. Closely related is the following conjecture of Waldhausen [W], which we will solve for genus two.

Conjecture 1. For each $g \in Z_{+}$, a closed 3-manifold has finitely many Heegaard splittings of genus $g$ up to homeomorphism.

Received by the editors July 3, 1990.

1980 Mathematics Subject Classification (1985 Revision). Primary 57M25.

Partially supported by NSF grant DMS-8823009 and by the Sloan Foundation. 
A Heegaard splitting of a closed orientable 3-manifold $M$ is a decomposition of $M$ into two handlebodies. More precisely, it is a triple $\left(F, H_{1}, H_{2}\right)$ where $F$ is an embedded connected orientable surface in $M, H_{1}$, and $H_{2}$ are handlebodies embedded in $M, M=H_{1} \cup H_{2}$, and $H_{1} \cap H_{2}=F=\partial H_{1}=\partial H_{2}$. $F$ is called a Heegaard surface. We say that two Heegaard splittings of $M$, $\left(F, H_{1}, H_{2}\right)$, and $\left(F^{\prime}, H_{1}^{\prime}, H_{2}^{\prime}\right)$, are equivalent if there is a homeomorphism $f: M \rightarrow M$ such that $f(F)=F^{\prime}, f\left(H_{1}\right)=H_{1}^{\prime}$, and $f\left(H_{2}\right)=H_{2}^{\prime}$, and similarly that $\left(F, H_{1}, H_{2}\right)$ and $\left(F^{\prime}, H_{1}^{\prime}, H_{2}^{\prime}\right)$ are equivalent up to isotopy if in addition $f$ is isotopic to the identity. Any closed orientable 3-manifold admits a Heegaard splitting. Of particular interest are the splittings with Heegaard surface of smallest possible genus. The genus of such a splitting is called the genus of $M$.

Let $M$ be a Riemannian 3-manifold and let $k$ be a positive integer. Let $E_{k}(M)$ denote the space of minimal embeddings of a surface of genus $k$ together with the space of minimal immersions of a surface of genus $k$, which double cover an embedded minimal nonorientable surface. Let $I_{k}(M)$ denote the space of minimal immersions of a surface of genus $k$. We work with the $C^{\infty}$-topology on the space of immersed minimal surfaces, so that a sequence of surfaces $\left\{S_{i}\right\}$ converges to $S$ if for each $p \in S$ there is a coordinate neighborhood $U_{p}$ of $p$ in $M$ such that each of the $S_{i}$ is represented by a smooth graph in $U_{p}$, converging smoothly to $S . E_{k}(M)$ is a closed subset of $I_{k}(M)$, since limits of smooth minimal embeddings are either embeddings or immersions that double cover an embedded surface.

The following lemma gives a compactness statement for the space of genus two and three minimal surfaces in certain manifolds. The proof of Lemma 2 is essentially contained in work of Uhlenbeck [U] and Choi-Schoen [CS].

Lemma 2. Let $M$ be a closed 3-manifold with negative sectional curvature.

(a) $E_{2}(M)$ and $I_{2}(M)$ are compact.

(b) If there does not exist an embedded nonorientable surface of genus 3 in $M$ then $E_{3}(M)$ is compact.

This should be compared to the results of Choi-Schoen, who showed that $E_{k}(M)$ is compact for all $k$ if $M$ is a closed simply connected 3-manifold with positive Ricci curvature. A similar argument also appears in [A, Corollary 4.3].

Before proving Lemma 2 we state a well-known compactness result.

Lemma 3. Let $\left\{S_{i}\right\}$ be a sequence of smooth immersed minimal surfaces of genus $g$ in a compact Riemannian manifold. Suppose that the area and second fundamental forms of $\left\{S_{i}\right\}$ are uniformly bounded. Then a subsequence converges smoothly to a minimal immersion.

Proof of Lemma 3. This standard result follows from Ascoli's theorem when the surfaces are expressed as graphs in local charts.

Proof of Lemma 2. Since $M$ is a closed 3-manifold with negative sectional curvature, there is a negative constant $K_{0}$ such that the sectional curvatures $K_{M}$ of $M$ satisfy $K_{M} \leq K_{0}$. Let $\left\{F_{i}\right\}$ be a sequence of minimal embeddings of genus $g$. Then the Gauss-Bonnet theorem states that $\int_{F_{1}} K=2 \pi(2-2 g)$ where $K$ is the curvature of the induced metric on $F_{i}$. Let $\lambda_{1}$ and $\lambda_{2}$ be 
the principle normal curvatures of $F_{i}$. The mean curvature $H$ is given by $H=\lambda_{1}+\lambda_{2}=0$, and $K$ is given by $K=K_{M}+\lambda_{1} \lambda_{2}$ by Gauss's theorem. We then have that $K=K_{M}+\lambda_{1} \lambda_{2} \leq K_{0}-\left(\lambda_{1}\right)^{2} \leq K_{0}<0$, so that

$$
2 \pi(2-2 g)=\int_{F_{i}} K \leq \int_{F_{i}} K_{0}=\operatorname{Area}\left(F_{i}\right) K_{0} \quad \text { and } \quad \operatorname{Area}\left(F_{i}\right) \leq 2 \pi(2-2 g) / K_{0} .
$$

It is proved in [CS] that an $L^{2}$-bound on the second fundamental form of a sequence of minimal surfaces $\left\{F_{i}\right\}$ implies that the pointwise norm of the second fundamental form is uniformly bounded away from a neighborhood of a finite number of isolated points, where handles are pinching off in the limit. This implies the existence of minimal immersions of smaller genus, which give rise to the original surfaces $F_{i}$ by a process of taking a number of copies of the boundary of a regular neighborhood and tubing them together with at least one tube. In negative curvature manifolds, no minimal immersions of nonnegative Euler characteristic exist, implying that the second fundamental form of a sequence of genus two surfaces $\left\{F_{i}\right\}$ is uniformly bounded. For genus three embeddings, we have to assume there is no minimal embedding homeomorphic to $P^{2} \sharp P^{2} \sharp P^{2}$ in $M$ to get the same conclusion, as the boundary of a regular neighborhood of such a surface is a genus two surface, which becomes genus three with the addition of a tube. So in each case we can conclude that the second fundamental form and the area of the sequence of surfaces $\left\{F_{i}\right\}$ are uniformly bounded, and so a subsequence converges to a minimal immersion by Lemma 3. A minimal surface that is the limit of a sequence of minimal embeddings is either embedded or a double covering of an embedding. This proves Lemma 2.

We now apply Lemma 2 to obtain a finiteness result for the number of Heegaard splittings of certain 3-manifolds.

Theorem 4. A closed 3-manifold $M$ has a finite number of genus 2-Heegaard splittings.

Proof. If the Heegaard genus of $M$ is zero or one, then the conclusion follows from the classification theorems of Waldhausen [W] and Bonahon-Otal [BO]. If the manifold is Haken, then Johannsen has recently shown that $M$ admits only finitely many Heegaard splittings [J], so we suppose that $M$ is non-Haken. Thurston's orbifold theorem [Th2] implies that any genus two 3-manifold has a decomposition along spheres and incompressible tori into pieces with geometric structure. Since $M$ is non-Haken it follows that there are no tori in the decomposition, and that $M$ is either hyperbolic, a Seifert fiber space with three exceptional fibers and orbit space the 2 -sphere, or a connect sum of two lens spaces.

If $M$ is a connect sum of two lens spaces, there exists an essential 2-sphere intersecting the Heegaard surface in one curve and splitting $M$ into two genus one manifolds, by a theorem of Haken [H]. This 2-sphere is unique up to homeomorphism by Milnor [M]. Bonahon showed that lens spaces have unique Heegaard splittings, and it follows that $M$ also has a unique splitting [B].

If $M$ is a Seifert fiber space with three exceptional fibers and orbit space the 2 -sphere, results of Boileau-Otal $[\mathrm{BoO}]$ show that there are only finitely many genus two splittings of $M$. 
The final case is that $M$ is hyperbolic. Given a Heegaard splitting, results of Pitts-Rubinstein [PR1, PR2] imply that either the Heegaard surface is isotopic to a minimal embedding or there is a smaller genus minimal embedding. The Gauss-Bonnet theorem implies that there are no minimal embeddings of genus zero or one in a negative sectional curvature manifold. Lemma 2 implies that only a finite number of surfaces can result from the first possibility, up to isotopy.

Note. The same methods can be used to show that a 3-manifold with a positive Ricci curvature metric admits only finitely many Heegaard splittings of genus zero, one or two, and with some additional argument, genus three. It follows from a theorem of Hamilton [Ha] that these manifolds are all Seifert fiber spaces with orbit space the 2-sphere and 3 exceptional fibers and so most of these results are covered by known theorems on the classification of Heegaard splittings of genus two Seifert fiber spaces [B, BO, BoO].

We next discuss another conjecture of Waldhausen to which the method developed above has some application [W].

Conjecture 5. Let $M$ be irreducible and $f: M \rightarrow M$ a diffeomorphism. If $f$ is homotopic to the identity then $f$ is isotopic to the identity.

This conjecture is known to hold for Seifert fiber spaces and for Haken manifolds, as well as in some other cases [W, Sc, BO]. It is false for reducible manifolds $[\mathrm{FW}]$. We will show that it is close to true when the manifold is genus two.

Theorem 6. An irreducible genus two manifold has a finite number of isotopy classes of diffeomorphisms homotopic to the identity.

Proof. If $M$ is Haken this is a result of Waldhausen, so we assume that $M$ is non-Haken. Since $M$ is irreducible and genus two, [Th2] implies that it is hyperbolic, as shown in Theorem 4. Let $\left\{h_{i}\right\}$ be an infinite sequence of diffeomorphisms of $M$, each homotopic to the identity. It suffices to show that two of the diffeomorphisms are isotopic. By passing to a subsequence we can assume that each $h_{i}$ leaves invariant a Heegaard surface $H$, since there are only finitely many such surfaces up to isotopy. Moreover we can find $h_{i}$ and $h_{j}$ such that $h=h_{j} h_{i}^{-1}$ does not interchange the handlebodies on either side of $H$. We will show that $h$ is isotopic to the identity.

A genus two Heegaard splitting defines an involution $\tau$ on $M$ with a 1dimensional fixed point set $\sigma$, and $\tau$ leaves $H$ invariant. The action of $\tau$ on $H$ lies in the center of the mapping class group of $H$, and thus commutes with $\left.h\right|_{H}$. Thus $h$ can be isotoped so that $h$ and $\tau$ commute on $H$. We now show that $h$ can be isotoped so that $h$ and $\tau$ commute on all of $M$. Since $h$ and $\tau$ commute on $H$, it suffices to show that a diffeomorphism $h$ of a handlebody $X$ to itself which commutes with $\tau$ on $H=\partial X$ can be isotoped rel boundary to commute with $\tau$ on $X$. To see this we pick a nonseparating $\tau$-invariant disk $E$ in $X$, so that $\tau E=E$. Then $h(E)$ is a disk in $X$ whose boundary $h(\partial E)$ satisfies $\tau h(\partial E)=h \tau(\partial E)=h(\partial E)$, so $h(\partial E)$ is invariant under $\tau . h(\partial E)$ bounds a $\tau$-equivariant disk $E^{\prime}$ isotopic to $h(E)$ and we can isotop $h$, rel boundary, so that $h(E)$ coincides with $E^{\prime}$ and so that $\tau$ and $h$ commute on $H \cup E$. Repeating on a second nonseparating $\tau$-invariant disk in $X$, we cut $H$ into a 3-ball so that $h$ and $\tau$ commute on the boundary of this 
ball. A $\tau$-equivariant map of a 2 -sphere to a 2 -sphere can be extended to a $\tau$ equivariant map of the 3-ball by coning. This extension defines an isotopic map which commutes with $\tau$ on $X$. Repeating on each of the two handlebodies, we arrive at an isotopy of $h$ on $M$ that commutes with $\tau$. It follows that, after an isotopy, $h$ leaves $\sigma$ pointwise fixed. By Thurston's orbifold theorem, a hyperbolic manifold with an involution having a 1-dimensional fixed point set admits a hyperbolic metric such that the diffeomorphism is a hyperbolic isometry, and the fixed point set is a hyperbolic geodesic. So the fixed point set of $\tau$ is a hyperbolic geodesic in a hyperbolic metric. Theorem 3.3 of [HS] states that if $M$ is a hyperbolic 3-manifold, $L$ an essential unlinked link in $M$ and $h$ a homeomorphism of $M$ that is homotopic to the identity and fixes $L$, then $h$ is isotopic to the identity. A hyperbolic geodesic is a special case of an essential unlinked link and it follows that $h$ is isotopic to the identity, concluding the proof of Theorem 6 .

Closely related to Conjecture 5 is:

Conjecture 7. Let $M$ be an irreducible non-Haken 3-manifold with infinite $\pi_{1}$. Then the mapping class group of $M$ is finite.

The mapping class group of $M$ is the group of diffeomorphisms modulo the equivalence of isotopy. Even for a hyperbolic manifold, it is not known whether this group is finite. It is known that $\operatorname{Out}\left(\pi_{1}(M)\right)$, the group of automorphisms of the fundamental group modulo inner automorphisms, is finite, but it is unknown whether these two groups coincide. Conjecture 7 holds for genus two manifolds.

Corollary 8. The mapping class group of a non-Haken irreducible infinite $\pi_{1}$ 3-manifold of genus two is finite.

Proof. Since $M$ is hyperbolic, Out $\left(\pi_{1}(M)\right)$ is finite by Mostow's Rigidity Theorem. The result then follows from Theorem 6.

\section{REFERENCES}

[A] M. Anderson, Curvature estimates for minimal surfaces in 3-manifolds, Ann. Sci. Ecole Norm. Sup. Ser. 18 IV (1985), 89-105.

[B] F. Bonahon, Diffeotopies des espaces lenticulaires, Topology 22 (1983), 305-314.

[BO] F. Bonahon and J. P. Otal, Scindements de Heegaard des espaces lenticulaires, Ann. Sci. Ecole Norm. Sup. (4) 16 (1983), 451-466.

[BoO] F. Boileau and J. P. Otal, Groupe des diffeotopies de certaines varietes de Seifert, C. R. Acad. Sci. Paris 303 (1986), 19-22.

[CS] H. Choi and R. Schoen, The space of minimal embeddings of a surface into a space of positive Ricci curvature, Invent. Math. 81 (1985), 387-394.

[FW] J. L. Friedman and D. M. Witt, Homotopy is not isotopy for homeomorphisms of 3manifolds, Topology 25 (1986), 35-44.

[H] W. Haken, Some results on surfaces in 3-manifolds, Studies in Modern Topology, Math. Assoc. Amer., Prentice Hall, 1968, pp. 34-98.

[Ha] R. Hamilton, Three-manifolds with positive Ricci curvature, J. Differential Geometry 17 (1982), 255-306.

[HS] J. Hass and P. Scott, Homotopy and isotopy of 3-manifolds, preprint.

[J] K. Johannsen, Heegaard surfaces in Haken 3-manifolds, Bull. Amer. Math. Soc. (N.S.) 23 (1990), 91-98. 
[M] J. Milnor, A unique factorization theorem for 3-manifolds, Amer. J. Math. 84 (1962), 1-7.

[PR1] J. Pitts and J. H. Rubinstein, Existence of minimal surfaces of bounded topological type in 3-manifolds, Proc. Centre Math. Anal. Austral. Nat. Univ. 10 (1986), 163-176.

[PR2] __ Applications of minimax to minimal surfaces and the topology of 3-manifolds, Bull. Amer. Math. Soc. 19 (1988), 303-309.

[S] M. Sakuma, Manifolds with infinitely many non-isotopic Heegaard splittings of minimal genus, preprint.

[Sc] P. Scott, There are no fake Seifert fibre spaces with infinite $\pi_{1}$, Ann. of Math. 117 (1983), 35-70.

[Th1] W. Thurston, The geometry and topology of 3-manifolds, Princeton Univ. Lecture Notes, 1978.

[Th2] __, An orbifold theorem for 3-manifolds, preprint.

[U] K. Uhlenbeck, Minimal embeddings of surfaces in hyperbolic 3-manifolds, preprint, 1981.

[W] F. Waldhausen, On some recent results in 3-dimensional topology, Proc. Sympos. Pure Math. 64 (1977), 21-38.

[Wh] B. White, The space of minimal submanifolds for varying Riemannian metrics, preprint, 1987.

Department of Mathematics, University of California, Davis, California 95616

E-mail address: hass@ucdmath.ucdavis.edu,jhass@ucdavis.bitnet 